\title{
Dissociation between controlled and automatic processes in the behavioural variant of fronto-temporal dementia
}

\author{
Fabienne Collette ${ }^{1,2,3}$, Martial Van der Linden ${ }^{1,4}$, Eric Salmon ${ }^{2,5}$ \\ ${ }^{1}$ Cognitive and Behavioral Neuroscience Centre, University of Liège, Liège, Belgium \\ ${ }^{2}$ Cyclotron Research Center, University of Liège, Liège, Belgium \\ ${ }^{3}$ Fonds National de la Recherche Scientifique, Belgium \\ ${ }^{4}$ Cognitive Psychopathology Unit, University of Geneva, Geneva, Switzerland \\ ${ }^{5}$ Memory Centre, Neurology Department, CHU, Liège, Belgium
}

Running head title: Impaired controlled processes in bv-FTD

* Correspondence concerning this article should be addressed to Fabienne Collette, Neuropsychology Unit, University of Liège, Boulevard du Rectorat 3 (B33), 4000 Liège, Belgium.

Telephone: (32) 43662274

Fax: (32) 43662875

E-mail: f.collette@ulg.ac.be 


\section{Abstract}

A decline of cognitive functioning affecting several cognitive domains was frequently reported in patients with frontotemporal dementia. We were interested in determining if these deficits can be interpreted as reflecting an impairment of controlled cognitive processes by using an assessment tool specifically developed to explore the distinction between automatic and controlled processes, namely the process dissociation procedure (PDP) developed by Jacoby [1]. The PDP was applied to a word stem completion task to determine the contribution of automatic and controlled processes to episodic memory performance and was administered to a group of 12 patients with the behavioural variant of frontotemporal dementia (bv-FTD) and 20 control subjects (CS). Bv-FTD patients obtained a lower performance than CS for the estimates of controlled processes, but no group differences was observed for estimates of automatic processes. The between-groups comparison of the estimates of controlled and automatic processes showed a larger contribution of automatic processes to performance in bv-FTD, while a slightly more important contribution of controlled processes was observed in control subjects. These results are clearly indicative of an alteration of controlled memory processes in bv-FTD.

Keywords: frontotemporal, dementia, executive functions, controlled processes, behaviour 


\section{Introduction}

The behavioural variant of fronto-temporal dementia (bv-FTD) constitutes, together with semantic dementia and progressive non-fluent aphasia, the group of lobar neurodegenerative diseases essentially involving the frontal and temporal lobes. In the behavioral variant of the disease, frontal atrophy is more prominent than temporal atrophy, and while the atrophy is generally bilateral, some reports suggest that the right hemisphere is more involved than the left $[2,3]$. Behavioural and personality disturbances constitute a major clinical characteristic of bv-FTD patients (for reviews, see $[4,5]$ ) and they concern changes in insight, affect modulation and social conduct [6, 7]. Cognitive impairment is not as pronounced as behavioural changes in the first stages of the disease, and many patients may perform within normal limits on traditional neuropsychological tests [8-10]. However, as the disease progresses, the number and severity of behavioural changes increase, and cognitive impairments emerge. At this stage, the most significant impairments are found on executive tasks, associated with variable memory performance, but only moderate deficits affecting language, constructional abilities and intelligence (IQ) have been reported (for reviews, see $[4,7,11]$ ).

Studies that compared verbal episodic memory functioning of these patients to healthy elderly subjects showed decreased performances for free recall tasks involving words or text, and for both immediate and delayed recall $[9,10,12,13]$. Recognition performance was usually preserved by comparison to recall performance $[7,12,13]$, but this was not observed in all studies $[14,15]$. The observed discrepancy in recognition performance is probably due to a differential involvement of recollection and familiarity processes according to the task characteristics (forced-choice versus yes/no test format, similarity between target and distracters...) [16]. According to these characteristics, the recognition task will require, in various proportions, automatic or controlled processes. Bv-FTD patients also benefited more from cues, had better encoding and demonstrated a slower forgetting rate than $A D$ patients $[12,13,17]$. Taken as a whole these data lead to the conclusion that the episodic memory changes seen in bv-FTD 
may be due in part to executive impairments such as a lack of active strategies for learning and retrieval of information $[13,18]$.

The assessment of the different subcomponents of working memory [19] showed mitigated results for both the automatic temporary storage systems (the phonological loop and visuospatial sketchpad) and the central executive, with a preserved performance by comparison to healthy elderly participants reported in some studies $[9,12,15]$ but not in others [13] [12]

Finally, a lower performance on tasks used to explore various aspects of executive functioning (Wisconsin Card Sorting Test, Trail Making test, Stroop task, verbal fluency, Hayling task, ...) was also frequently reported in patients with bv-FTD (e.g. $[9,12,15,20-23])$. These executive deficits appear more important in patients with widespread frontal-lobe atrophy extending into the dorsolateral frontal cortex. By contrast, patients with relatively restricted medial and orbital frontal-lobe atrophy may perform surprisingly well on executive tasks, despite gross behavioural changes [24-26].

As a whole, these studies showed the presence of impairments in the domains of episodic memory (for recall tasks mainly) and executive functioning, while short-term memory appears relatively preserved in bv-FTD. However, most of these studies were performed in a clinical perspective in order to improve differential diagnosis between bv-FTD and other neurodegenerative conditions $(e . g,[15,21,27]$ and were not designed to explore specific hypotheses concerning the cognitive functioning of these patients (see however $[12,13,28])$.

Consequently, the objective of the present study was to explore if the distinction proposed between automatic and controlled cognitive processes can be applied to the pattern of impairments observed in bv-FTD patients. According to Hasher and Zacks [29], automatic processes are notably characterized by a minimal involvement of attentional resources and an inflexible and unconscious way of functioning. On the contrary, controlled processes require more attentional resources, are conscious, flexible and likely 
to improve with practice. More particularly, we were interested to determine if bv-FTD patients present a specific impairment of controlled processes in association to a preservation of automatic ones by using an assessment tool specifically developed to explore the distinction between automatic and controlled aspects of cognition, namely the process dissociation procedure (PDP) developed by Jacoby [1]. The PDP allows a quantitative estimation of the contribution of controlled and automatic processes involved in the same cognitive task. This procedure was more particularly used in the episodic memory domain and involves the comparison of performance when controlled and automatic processes act in a convergent way to the performance, and when these processes act in an opposite way. In the present study, the PDP was applied to a word stem completion task. As the complementary testing battery also comprised a series of executive and non-executive tasks and the lowa Scale of Personality Changes (ISPC; [30], assessing socio-emotional changes consecutive to brain damages), correlation analyses were performed to explore the relationships in bv-FTD patients between specific cognitive abilities/behavioural measures and the estimates of controlled aspects of cognition assessed by the PDP.

\section{Method}

\subsection{Participants}

Nineteen patients initially referred as suffering from frontotemporal dementia (FTD; 3 women) participated in the present study. The diagnostic of FTD was established by an experienced neurologist (ES) according to consensual criteria for FTD [31] based on (1) the demographic information and clinical history obtained during an interview with the patient and a caregiver, and (2) neurological and general examinations. To perform the diagnosis, the clinician had access to recent anatomical neuroimaging and laboratory data, as well as a complete assessment of cognitive functioning performed by a neuropsychologist. Twelve patients ( 2 women) were subsequently selected on the basis of a two-year 
follow-up that confirmed the initial diagnosis of FTD. The FTD patients showed the behavioural presentation of the disease (the frontal variant or bv-FTD); cases with specific characteristics of semantic dementia and primary progressive aphasia were excluded from this study. The patients' ages ranged from 56 to 80 years (mean age: $67.5 \pm 8.6$ years), their mean performance at the MMSE was $23.4 \pm 4.9$ and the disease duration varied between one and five years.

The selection procedure can be summarized in the following way. The patients were first referred to the experienced neurologist (ES) by hospital neurologists for 18FDG PET-scan and research neuropsychological examinations. The clinical diagnosis of FTD was confirmed using established criteria at this time [41]. The patients were then submitted to the battery of cognitive tasks reported here. The importance of a confirmed diagnosis in studies on FTD was recently emphasized by Kipps et al. [32] who reported that some cases initially meeting current criteria for FTD may not develop a neurodegenerative syndrome. Consequently, medical files of the patients were re-examined after two years to confirm the initial diagnosis and remove from our sample of bv-FTD patients those who remained stable during follow-up.

The patient group was matched for age and education level to a group of 20 elderly volunteers (4 women) with no history of alcoholic abuse, neurological problems or psychiatric disorders. They were recruited from the dwelling community. Each control subject performed above the cut-off score of 130 at the Mattis dementia rating scale [33]. A slightly higher than usual cut-off score was used in order to increase the sensitivity and specificity of the inclusion/exclusion criteria [34].

The control subjects did not differ from FTD patients according to age $[t(30)=0.55 p>0.5]$ and education level $[t(30)=0.35, p>0.5]$. Overall performance on the Mattis Dementia Rating Scale was significantly lower for FTD patients than for control subjects $[t(30)=-6.27, p<0.0001]$, indicating mild to moderate level of global cognitive deficit in the bv-FTD patients. With regard to depression scales, there was no significant differences between the two groups for the total scores on the short version of the Geriatric 
Depression Scale (GDS; [35]) and the Hospital Anxiety and Depression scale (HAD; [36]) [respectively, $t(29)=1.70, p=0.1 ; t(28)=0.97, p>0.1]$. The scores on the HAD anxiety and depression subscales were also similar between the two groups [respectively, $t(28)=0.22, p>0.5 ; t(28)=1.52, p>0.1$ ].

All subjects were native French-speakers. Experimental procedures were done in accord with the Helsinki Declaration of 1975. The study was approved by the Ethic Committee of the University Hospital in Liège and informed consent was obtained in all patients and control subjects. Demographic data of the participants are presented in Table 1.

[Insert Table 1]

\subsection{Experimental tasks}

\subsubsection{Exploration of controlled and automatic cognitive processes using the Process Dissociation} Procedure (PDP; [1])

The material comprised 96 six-letter French words. This list of stimuli was created such as each word stem (i.e., the first three letters of each word) was unique within the experiment but not within the language. Each word stem could be completed by at least five six-letter French words (e.g., cha - - - : chaque, chacun, chaton, chacal chatte, etc), but only one of the completions appeared in the experiment. Words were distributed between two conditions (inclusion and exclusion) and different lags $(0,3,12)$ between presentation of the one word and its corresponding stem. These lags allowed to vary the task difficulty by inducing retention intervals of various length. For both conditions, 8 words were presented at lag 0 (to check that patients complied with instructions), and 16 words at lag 3 and 12 . Sixteen words were also used as baseline. Baseline items corresponded to stems presented for completion without prior presentation of the target item and allowed to obtain a score for random completion of a stem by the experimental word. Words and stems were intermixed in each condition. The list order in the inclusion and exclusion condition was pseudo-random with the restriction than no 
more than two words of a given type (lags 0,3 or 12 ) could occur consecutively throughout the experiment and all conditions were distributed evenly across the test list (see Figure 1).

\section{[Insert Figure 1]}

The test comprised two separate conditions (inclusion and exclusion). Condition order was counterbalanced across subjects (half of the subjects began with the inclusion condition and the other half with the exclusion condition). For both conditions, the procedure was the same (except that the stimuli were different); only the instructions on how to complete the stems were different. Each condition involved the intermixed presentation of words and stems. Each six-letter word was presented for $3 \mathrm{~s}$, followed by $0.5 \mathrm{~s}$ of black screen. Participants were asked to read the words aloud and to remember them for a subsequent memory test. Stems were presented for $15 \mathrm{~s}$ and participants had to complete them according to two different sets of instructions (inclusion and exclusion instructions).

In the inclusion condition, subjects were told to complete the stem with a word that had been presented in the test list. If they could not think of an old word, they were asked to complete the stem with the first six-letter word that came to mind. In the exclusion condition, participants were asked to avoid completing the stem with a previously studied word, but to give a new word. That is, they had to reject the studied word and name another six-letter word that would complete the stem. If subjects could not remember the learned word, they had to give the first appropriate six-letter word that came to mind to complete the stem.

Furthermore, each word stem was preceded by the presentation of either the prompt "old" (in the inclusion condition) or the prompt "new" (in the exclusion condition) centred two lines above the word stem in light blue capital letters (in the inclusion condition) or light red capital letters (in the exclusion condition). The prompt was presented $3 \mathrm{~s}$ prior to the presentation of the word stem and remained on the screen with the word stem until the participant responded or until the 15-s period had 
elapsed. During these $3 \mathrm{~s}$, the experimenter briefly repeated the instructions, saying in the inclusion condition, "you have to complete the stem with a word seen earlier or, if you cannot, with the first sixletter word that comes to mind," and in the exclusion condition, "don't forget, you have to remember the words you studied, reject them, and think of another six-letter word to complete the stem." The repetition of prompts and instructions was intended to prevent the subjects from forgetting the instructions, and also to prevent perseveration (applying the instructions for the first condition to the second condition).

Completion rule. In all conditions, subjects had to complete stems according to the following rules: no plurals, no proper nouns, and no conjugated verbs (except past participles). If the subject came up with a solution that met these criteria, the experimenter pressed the space bar and the next item appeared after the presentation of a black screen for $0.5 \mathrm{~s}$. Otherwise, the experimenter informed the subject of the error and the subject was encouraged to generate a more appropriate solution. Subjects had a maximum of $15 \mathrm{~s}$ to complete each stem. If the stem had not been completed with an appropriate six-letter word when the allotted time was over, the stem disappeared automatically and was replaced by a black screen for $0.5 \mathrm{~s}$ before the presentation of the next item.

Baseline condition. Before the inclusion and exclusion conditions, 16 stems were presented for completion without prior presentation of the target item. Subjects were informed to give the first sixletter word that came to mind. This baseline condition was designed to determine the probability that a subject would complete a stem with the chosen target word without having seen it. This probability represents the base-rate level of completion for stems. The aim of this control condition is to verify that automatic processes actually corresponded to the unconscious influence of memory by showing that estimates of automatic processes are significantly above the base-rate or chance level.

Practice session. Before each test condition, a practice session (comprising three stimuli and their corresponding stems) was administered to make subjects familiar with the prompts, stimuli, and 
instructions. This practice session was repeated if necessary until the subject understood the task.

Estimates of controlled and automatic processes. In the inclusion condition, subjects were asked to complete a stem with a previously studied word or, if they were unable to do so, to use the first word that came to mind. Therefore, in this condition, subjects could correctly complete a stem with an earlier studied word either because they consciously recollected having seen the word before (C), or because it was the first word that came to mind automatically (A), without any recollection that the word had been presented earlier $(1-\mathrm{C})$. Thus, the probability of completing a stem in the inclusion condition can be represented as: Inclusion $=\mathrm{C}+\mathrm{A}(1-\mathrm{C})$. By contrast, in the exclusion condition, subjects were asked to complete the stem with a new word that had not been encountered during the study phase and to avoid (exclude) words that had been studied. In this condition, then, subjects might incorrectly complete a word that had been studied earlier only if that word came automatically to mind (A), without any controlled recollection that it had been presented earlier $(1-\mathrm{C})$. Thus, the probability of producing an error (i.e., completing a stem with a word that had been studied earlier) in the exclusion condition can be represented as: Exclusion $=\mathrm{A}(1-\mathrm{C})$. Following Jacoby (1991), the contribution of controlled processes in the task can be estimated by subtracting the probability of responding with a studied word in the exclusion condition from the probability of responding with an old (i.e., studied) word in the inclusion condition. Once an estimate of controlled processes has been obtained, the contribution of automatic processes corresponds to the probability of completing a stem with the studied word in the exclusion condition divided by one minus the probability of completing a stem with the studied word in the inclusion condition.

\subsubsection{Brief complementary cognitive and behavioral assessment}

Several other tasks were proposed to characterize (1) executive functioning (Stroop [37] and Hayling [38] tasks, verbal (phonemic and semantic) fluency tasks, Trail Making test [39], delayed alternation task 
[40] and Brixton task [41]), (2) short term memory processes (assessment of the phonological store and articulatory rehearsal process [42] of the phonological loop) and (3) speed of processing (letter comparison task [43], Stroop color naming condition and Stroop word reading condition [37]). The behavioural changes were assessed with the lowa Scales of Personality Changes (ISPC, [30]). More precisely, we were interested in characterizing socio-affective changes according to the higher-order psychopathology of internalizing (Depression, Anxiety, and Social Withdrawal subscales) and externalizing (Irritability, Impulsivity, Lack of Planning, Insensitivity, Social Inappropriateness, Impatience, Aggression, and Inappropriate Affect subscales) dimensions (see [44]).

\subsection{Statistical analyses}

Between groups comparisons were performed using ANOVAs with group as independent variable (control vs. bv-FTD) and the different cognitive measures as dependant variable. A statistical level of $p<$ 0.05 was used for all analyses. The effect size of each ANOVA was reported as partial eta square (ES). Partial eta square is generally interpreted as the proportion of variance of the dependent variable that is related to the factor. Traditionally, eta square values of $.01, .06$, and .14 represent small, medium and large effect sizes, respectively [45]. Due to the relatively small sample size in the bv-FTD group, we have also performed non-parametric analyses (Mann-Wihtney $U$ and Sign tests). Similar results were obtained, except for two measures that became non-significant with non-parametric tests. For these measures, non-parametrical values were also reported in the result section. Non-parametric correlations (spearman-rank test, $p<0.05$ ) were performed in the group of bv-FTD to determine the presence of associations between measures of controlled/automatic memory processes and other measures of cognitive/behavioural functioning. 


\section{Results}

3.1 Exploration of controlled and automatic cognitive processes using the Process Dissociation Procedure (PDP; [1])

Table 2 summarizes the scores for control participants and bv-FTD patients in each experimental condition (Lags 0,3 , and 12) as well as the estimates of controlled and automatic processes.

[Insert Table 2 near here]

\section{Word stem completion}

The proportion of stems completed with the target word in the inclusion condition was analysed with a 2 (group: controls vs FTD) X 3 (lag interval: lag 0 vs lag 3 vs lag 12) ANOVA with repeated measures on the last factor. The results showed a main group effect $[F(1,28)=13.93, p<0.0001$, partial $E S=0.33]$, with the control group producing more target words than the FTD patients; there was also a main effect for lag interval $[F(2,56)=100.15, p<0.0001$, partial $E S=0.78]$, with performance declining as the lag interval increased; however there was no significant interaction $[F(2,56)=2.47, p=0.09$, partial ES $=0.08]$, suggesting that the decrease in performance with the increase in the retention interval was similar in bv-FTD patients and control subjects. However, due to the small sample size, this absence of interaction should be replicated in further studies since the presence of a larger performance decline according to the interval in the bv_FTD group cannot be totally excluded on the basis of $p$ and effect size values reported here. In the exclusion condition, the analyses showed a main effect of group $[F(1,28)=12.48$, $\mathrm{p}<0.005$, partial ES $=0.30]$, with the bv-FTD patients producing more errors than the control group; a main effect for lag interval $[F(2,56)=24.37, p<0.0001$, partial $E S=0.46]$, with more errors produced as the interval increased; but no interaction effect $[F(2,56)=0.67, p=0.51$, partial $E S=0.02]$.

\section{Estimate of controlled processes}

The estimates of controlled processes were analyzed with a 2 (group: control vs. FTD) X 3 (lag interval: 
lag 0 vs. lag 3 vs. lag 12) ANOVA with repeated measures on this factor. The results revealed a main effect for group $[F(1,28)=19.92, p<0.0001$, partial $E S=0.41, F T D<C S]$, a main effect for lag interval $[F(2,56)=89.18, p<0.0001$, partial $E S=0.76 ; \operatorname{lag} 0>\operatorname{lag} 3>\operatorname{lag} 12]$ but no interaction between group and interval $[F(2,56)=1.39, p=0.26$, partial $E S=0.05]$.

\section{Estimate of automatic processes}

Considering the ceiling and floor effects for both groups in the inclusion and exclusion conditions at lag 0 (see Table 2), estimates of automatic processes were calculated only for Lags 3 and 12 . The estimates of automatic processes were analyzed with a 2 (group: control vs.FTD) X 2 (lag interval: lag 3 vs. lag 12) ANOVA with repeated measures on this factor. The results did not reveal significant effect for group $[F(1,28)=0.19, p=0.66$, partial $E S=0.66]$, nor for lag interval $[F(1,28)=2.16, p=0.15$, partial $E S=0.07]$ and no significant group versus lag interval interaction $[F(1,28)=0.19, p=0.67$, partial $E S=0.007]$.

\section{Comparison of the controlled and automatic processes estimates}

Finally, a between-group comparison of the controlled and automatic processes estimates (mean value of lags 3 and 12) was performed. A 2 (group: control vs.FTD) X 2 (process: controlled vs. automatic) ANOVA revealed a significant group effect $[F(1,28)=13.58, p<0.001$, partial $E S=0.33]$, no process effect $[F(1,28)=2.87, p=0.10$, partial $E S=0.09]$ and significant group $X$ process interaction effect $[F(1,28)=16.91$, $p>0.0005$, partial ES $=0.38]$. The interaction effect showed a larger contribution of automatic processes to performance in bv-FTD patients $(p<0.005)$ but a slightly more important $(p=0.056)$ contribution of controlled processes in control subjects. However, this tendency disappeared with a non-parametric analysis (Sign test: $Z(19)=1.61, p=0.11$ ).

\subsection{Brief complementary cognitive and behavioural assessment}

Results of group comparisons for the complementary cognitive tasks and behavioural assessment are 
presented in Tables 3, 4 and 5. As a whole, results are indicative of impaired executive processes (to note that the between-group difference for the number of errors in the interference condition of the Stroop task disappeared when non-parametric analyses were performed $[H(1,30)=2.36, p=0.12])$, associated to a preservation of the phonological loop of working memory, a normal processing speed and the presence of socio-emotional changes after the beginning of the disease. However, the RTs betweengroup comparison for the letter matching and naming Stroop tasks showed a statistical $p$ value of .09 and medium effect sizes. Consequently, these results should be replicated with a larger sample of bv_FTD and control participants to conclude that there exists no slowing down of information processing in the disease.

[Insert Tables 3, 4, 5 near here]

\subsection{Correlation analyses}

Non-parametric correlations (spearman-rank test, $p<0.05$ ) were performed in the group of bv-FTD to determine the presence of associations between measures of controlled/automatic memory processes and other measures of cognitive/behavioral functioning. The only significant correlation observed was between the estimate of controlled processes at the Jacoby task (lag 3 and 12) and the performance at the Brixton task ( $r=.67)$. Interestingly, automatic processes that might hypothetically be contaminated by a lack of inhibition in stem completion were not correlated to performances in conceptual inhibition (measured by response times and semantic relatedness of the response furnished in part B of the Hayling task).

\section{Discussion}

In this study, we assessed the performance of a group of bv-FTD patients (whose initial diagnosis was 
confirmed after a two-year follow-up; see [32]) on a word stem completion task encompassing automatic and controlled aspects of cognition using the Processes Dissociation Procedure [1]. On this basis, we were interested to determine if bv-FTD patients present a specific impairment of controlled cognitive processes, associated to a preservation of automatic ones.

The between-groups comparison of the estimates of controlled and automatic processes showed a larger contribution of automatic processes to performance in bv-FTD, while a slightly more important contribution of controlled processes was observed in control subjects. As a whole, these results suggest that memory deficits in bv-FTD come from a difficulty to spontaneously use efficient research strategy of information stored in memory, rather than a rapid decay of stored information per se. Indeed, the previously encoded information is accessed in a similar way in the two groups of participants when automatic processes intervene but not when the instruction emphasizes explicit search strategy of the information. In agreement with the interpretation of a reduced contribution of strategic processes to memory retrieval, several studies previously reported a better (or even preserved) performance on recognition $([7,12,13])$ or cued recall tasks $([12,13])$ by comparison to free recall tasks.

Otherwise, the normal performance of bv-FTD patients at lag 0 in the exclusion condition and the absence of correlation between measures of automatic processes (lags 3 and 12) and inhibition (RTs and response accuracy on the Stroop and Hayling tasks) indicates that inhibitory deficits are not the main determinants of the pattern of performance on the PDP, and more particularly that the performance in the exclusion task is not driven by an inability to suppress a dominant response (the word previously presented), as observed in the Hayling task (for a similar discussion in the context of normal aging, see Hay and Jacoby [46]). Similarly, the absence of correlation between PDP inclusion or automatic scores and Hayling or Stroop performance (RTs and accuracy) suggests that decreased inhibition did not explain automatic completion of words in bv-FTD. Interestingly, the higher percentage of stem completion in the exclusion condition is reminiscent of the "hyper-priming" sometimes reported in FTD patients [13] 
and a strong dependence on automatic processes might be a characteristic of bv-FTD.

Impairment of controlled processes appears also very sensitive to the early stages of Alzheimer's disease and might not allow to clearly distinguish between bv-FTD and Alzheimer's patients. Indeed, the contribution of controlled processes to memory performance (estimated by the PDP; [1]) was also decreased in Alzheimer patients [47, 48]. However, these two groups of patients may achieve similar scores on these tasks for different underlying reasons. Indeed, in the context of the PDP, we noticed that a large proportion of bv-FTD (8 out 12) presents a negative score for the estimate of controlled processes at the lags 3 or 12 . Such a pattern of results was not observed in our control participants nor in a group of patients with Alzheimer's disease and their matched controls examined in a previous study ([47], data analyzed but not presented in the published paper). Interestingly, the presence of negative scores in our bv-FTD group is not related to inhibitory dysfunction (as indicated by the absence of correlations between these scores and performance on the Hayling and Stroop tasks). A possible explanation is that the active search of a previously encoded information in bv-FTD counteracts the effect of automatic retrieval processes, possibly due to the use of inadequate monitoring strategies. For example, the automatic retrieval of previously encoded information could be systematically considered as false alarms in bv-FTD patients while the retrieval of information on the basis of automatic memory processes would not be associated to such monitoring processes in Alzheimer disease. These observations obviously need confirmation in further studies directly comparing performance of Alzheimer and FTD patients but could possibly provide another measure allowing to distinguish these neurodegenerative conditions.

Correlations analyses demonstrated few relationships between measures of controlled processes assessed by the PDP and executive tasks (the only significant correlation was found between controlled processes in the PDP and performance on the Brixton task). These results seem to indicate that controlled processes (as assessed by a word stem completion memory task) and executive processes does not represent exactly the same cognitive constructs, even if both depends on high-level cognitive 
processes. These results can be related to those obtained with group studies which employed a large battery of widely used executive tasks to examine executive functioning in several target populations, including normal young adults [49], normal elderly adults [50,51] and brain-damaged patients [52-54]. A highly consistent pattern of results across these studies is that the intercorrelations among the different executive tasks are low and are often not statistically significant, indicating the non-unitary nature of executive functioning. Even if the absence of correlations in the present study must be considered cautiously due to the small number of patients, results obtained are a supplementary argument in agreement with the non-unitary nature of these aspects of high-level cognition.

To summarize, the neuropsychological pattern of a group of patients with bv-FTD was characterized by impaired controlled but preserved automatic cognitive processes. Such a dissociation is not specific for bv-FTD, since it was previously reported in early AD [47]. However, qualitative analyses of performance could help to differentiate these pathologies. Otherwise, no relationship was found between behavioural and automatic or controlled processes in episodic memory, indicating that neuropsychological assessment setting could not evidence difficulties encountered in daily life and reported by relatives. 


\section{Acknowledgments}

This research was supported by the Belgian National Fund for Scientific Research (FNRS), the Interuniversity Attraction Poles Program P6/29, and the University Hospital in Liège. FC is Senior Researcher at the FNRS. There is no potential conflicts concerning the publication of this article 


\section{References}

[1] Jacoby LL (1991) A process dissociation framework: Separating automatic from intentional uses of memory. Journal of Memory and Language 30, 513-541.

[2] Perry RJ, Graham A, Williams G, Rosen H, Erzinclioglu SW, Weiner M, Miller B, Hodges J (2006) Patterns of frontal lobe atrophy in frontotemporal dementia: A volumetric MRI study. Dementia and Geriatric Cognitive Disorders 22, 278-287.

[3] Rosen HJ, Gorno-Tempini ML, Goldman WP, Perry RJ, Schuff N, Weiner M, al e (2002) Patterns of brain atrophy in frontotemporal dementia and semantic dementia. Neurology 58, 198-208.

[4] Grossman M (2002) Frontotemporal dementia: A review. Journal of the International Neuropsychological Society 8, 566-583.

[5] Neary D, Snowden JS, Mann DM (2005) Frontotemporal dementia. Lancet Neurology 4, 771-780.

[6] Bozeat S, Gregory CA, Lambon Ralph MA, Hodges JR (2000) Which neuropsychiatric and behavioural features distinguish frontal and temporal variants of frontotemporal dementia from Alzheimer's disease? Journal of Neurology, Neurosurgery and Psychiatry 69, 178-186.

[7] Harciarek M, Jodzio K (2005) Neuropsychological differences between frontotemporal dementia and Alzheimer's disease: A review. Neuropsychology Review 15, 131-145.

[8] Diehl-Schmid J, Pohl C, Perneczky R, Forstl H, Kurz A (2006) Behavioral disturbances in the course of frontotemporal dementia. Dementia and Geriatric Cognitive Disorders 22, 532-537.

[9] Hodges JR, Patterson K, Ward R, Garrard P, Bak T, Perry R, Gregory C (1999) The differentiation of semantic dementia and frontal lobe dementia (temporal and frontal variants of frontotemporal dementia) from early Alzheimer's disease: A comparative neuropsychological study. Neuropsychology 13, 31-40.

[10] Kramer JH, Jurik J, Sha SJ, Rankin KP, Rosen HJ, Johnson JK, Miller BL (2003) Distinctive neuropsychological patterns in frontotemporal dementia, semantic dementia, and Alzheimer disease. Cogn Behav Neurol 16, 211-218.

[11] Wittenberg D, Possin KL, Rascovsky K, Rankin KP, Miller BL, Kramer JH (2008) The early neuropsychological and behavioral characteristics of frontotemporal dementia. Neuropsychology Review 18, 91-102.

[12] Matuszewski V, Piolino P, de la Sayette V, Lalevee C, Pelerin A, Dupuy B, Viader F, Eustache F, Desgranges B (2006) Retrieval mechanisms for autobiographical memories: insights from the frontal variant of frontotemporal dementia. Neuropsychologia 44, 2386-2397.

[13] Pasquier F, Grymonprez L, Lebert F, Van der Linden M (2001) Memory impairment differs in frontotemporal dementia and Alzheimer's disease. Neurocase 7, 161-171.

[14] Pachana NA, Brauer Boone K, Miller BL, Cummings JL, Berman N (1996) Comparison of neuropsychological functioning in Alzheimer's disease and frontotemporal dementia. Journal of the International Neuropsychological Society 2, 505-510.

[15] Perry RJ, Hodges JR (2000) Differentiating frontal and temporal variant frontotemporal dementia from Alzheimer's disease. Neurology 54, 2277-2284.

[16] Bastin C, Van der Linden M (2003) The contribution of recollection and familiarity to recognition memory : A study of the effects of test format and aging. Neuropsychology 17, 14-24.

[17] Soderlund H, Black SE, Miller BL, Freedman M, Levine B (2008) Episodic memory and regional atrophy in frontotemporal lobar degeneration. Neuropsychologia 46, 127-136.

[18] Glosser G, Gallo JJ, Clark CM, Grossman M (2002) Memory encoding and retrieval in frontotemporal dementia and Alzheimer's disease. Neuropsychology 16, 190-196.

[19] Baddeley AD (1986) Working memory, Clarendon Press, Oxford.

[20] Collette F, Amieva H, Adam S, Hogge M, Van der Linden M, Fabrigoule C, Salmon E (2007) 
Comparison of inhibitory functioning in mild Alzheimer's disease and frontotemporal dementia. Cortex 43, 866-874.

[21] Giovagnoli AR, Erbetta A, Reati F, Bugiani O (2008) Differential neuropsychological patterns of frontal variant frontotemporal dementia and Alzheimer's disease in a study of diagnostic concordance. Neuropsychologia 46, 1495-1504.

[22] Gregory C, Lough S, Stone V, Erzinclioglu S, Martin L, Baron-Cohen S, Hodges JR (2002) Theory of mind in patients with frontal variant frontotemporal dementia and Alzheimer's disease: theoretical and practical implications. Brain 125, 752-764.

[23] Lough S, Kipps CM, Treise C, Watson P, Blair JR, Hodges JR (2006) Social reasoning, emotion and empathy in frontotemporal dementia. Neuropsychologia 44, 950-958.

[24] Gregory CA, Serra-Mestres J, Hodges JR (1999) Early diagnosis of the frontal variant of frontotemporal dementia: how sensitive are standard neuroimaging and neuropsychologic tests. Neuropsychiatry, Neuropsychology and Behavioral Neurology. 12, 128-135.

[25] Neary D, Snowden JS, Northen B, Goulding P (1988) Dementia of frontal lobe type. Journal of Neurology, Neurosurgery and Psychiatry 51, 353-361.

[26] Snowden JS, Neary D, Mann DMA (1996) Fronto-temporal lobar degeneration: Fronto-temporal dementia, progressive aphasia, semantic dementia, Churchill Livingstone, New York.

[27] Rascovsky K, Salmon DP, Ho GJ, Galasko D, Peavy GM, Hansen LA, Thal U (2002) Cognitive profiles differ in autopsy-confirmed frontotemporal dementia and AD. Neurology 58, 1801-1808.

[28] Piolino P, Chetelat G, Matuszewski V, Landeau B, Mezenge F, Viader F, de la Sayette V, Eustache $F$, Desgranges B (2007) In search of autobiographical memories: A PET study in the frontal variant of frontotemporal dementia. Neuropsychologia 45, 2730-2743.

[29] Hasher L, Zacks R (1979) Automatic and effortful processes in memory. Journal of Experimental Psychology: General 108, 356-388.

[30] Barrash J, Anderson SW, Jones RD, Tranel D (1997) in 25th Annual Meeting of the International Neuropsychological Society (Orlando, FL).

[31] Neary D, Snowden JS, Gustafson L, Passant U, Stuss D, Black S, Freedman M, Kertesz A, Robert $\mathrm{PH}$, Albert M, Boone K, Miller ML, Cummings J, Benson DF (1998) Frontotemporal lobar degeneration. A consensus on clinical diagnostic criteria. Neurology 51, 1546-1554.

[32] Kipps CM, Nestor PJ, Fryer TD, Hodges JR (2007) Behavioural variant frontotemporal dementia: not all it seems? Neurocase 13, 237-247.

[33] Mattis S (1973) (NFER-Nelson, Windsor).

[34] Monsch AU, Bondi MW, Salmon DP, Butters N, Thal L, Hansen LA, Wiederholt WC, Cahn DA, Klauber MR (1995) Clinical validity of the Mattis dementia rating scale in detecting dementia of the Alzheimer type. Archives of Neurology 52, 899-504.

[35] Yeasavage JA, Brink TL, Rose TL, Lum O (1983) Development and validation of a geriatric depression screening scale: A preliminary report. Journal of Psychiatric Research 17, 37-49.

[36] Zigmond AS, Snaith RP (1983) The hospital anxiety and depression scale. Acta Psychiatr Scand 67, 361-370.

[37] Stroop JR (1935) Studies of interference in serial verbal reactions. Journal of Experimental Psychology 6, 643-661.

[38] Burgess PW, Shallice T (1996) Response suppression, initiation and strategy use following frontal lobe lesions. Neuropsychologia 34, 263-273.

[39] Reitan RM, Wolfson D (1995) Category test and trail making test as measures of frontal lobe functions. The Clinical Neuropsychologist 9, 50-56.

[40] Freedman M, Oscar-Berman M (1986) Bilateral frontal lobe disease and selective DR deficits in humans. Behavioral neuroscience 100, 337-342.

[41] Burgess PW, Shallice T (1996) Bizarres responses, rule detection and frontal lobe lesions. Cortex 
32, 241-259.

[42] Belleville S, Peretz I, Malenfant D (1996) Examination of the working memory components in normal aging and in dementia of the Alzheimer type. Neurospychologia 34, 195-207.

[43] Salthouse TA, Babcock RL (1991) Decomposing adult age differences in working memory. Developmental Psychology 27, 763-776.

[44] Rochat L, Ammann J, Mayer E, Annoni J-M, Van der Linden M (2009) Executive disorders and perceived socio-emotional changes after traumatic brain injury. Journal of Neuropsychology 3, 213-227.

[45] Cohen J (1988) Statistical power analysis for the behavioral sciences, Lawrence Erlbaum Associates, Hillsdale,NJ.

[46] Hay JF, Jacoby LL (1999) Separating habits and recollection in young and older adults: Effects of elaborative processing and distinctiveness. . Psychology and Aging 14, 122-134.

[47] Adam S, Van der Linden M, Collette F, Lemauvais L, Salmon E (2005) Further exploration of controlled and automatic processes in early Alzheimer's disease. Neuropsychology 19, 420-427.

[48] Koivisto M, Portin R, Seinela A, Rinne J (1998) Automatic influences of memory in Alzheimer's disease. Cortex 34, 209-219.

[49] Lehto J (1996) Are executive function tests dependent on working memory capacity? The Quarterly Journal of Experimental Psychology 49A, 29-50.

[50] Lowe C, Rabbit P (1997) Cognitive models of aging and frontal lobe deficits. In Methodology of frontal and executive functions., Rabbitt $\mathrm{P}$, ed. Psychology Press, Hove, pp. 39-59.

[51] Robbins TW, Owen AM, Sahakian BJ, Lawrence AD, McInnes L, Rabbitt PMA (1998) A study of performance on tests from the CANTAB battery sensitive to frontal lobe dysfunction in a large sample of normal volunteers: implications for theories of executive functioning and cognitive aging. Journal of International Neuropsychological Society 4, 474-490.

[52] Burgess PW (1997) Theory and methodology in executive function research. In Methodology of frontal and executive functions, Rabbit P, ed. Psychology Press, Hove, pp. 81-116.

[53] Burgess PW, Alderman N, Evans J, Emslie H, Wilson BA (1998) The ecological validity of tests of executive functions. Journal of International Neuropsychological Society 4, 547-558.

[54] Duncan J, Johnson R, Swales M, Freer C (1997) Frontal lobe deficits after head injury: Unity and diversity of function. Cognitive Neuropsychology 14, 713-741. 


\section{Figure legends}

Figure 1. Example of a stimuli sequence

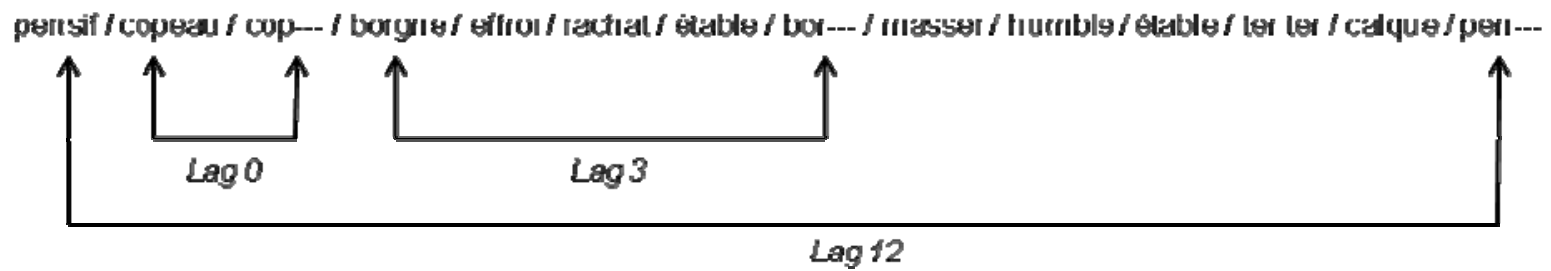


Table 1. Demographic data (mean and standard deviation) of the two groups of participants

\begin{tabular}{|l|l|l|}
\hline & Controls subjects & FTD patients \\
\hline Number of participants & 20 & 12 \\
\hline Age & $65.7(9.1)$ & $67.5(8.6)$ \\
\hline Gender (men/women) & $16 / 4$ & $10 / 2$ \\
\hline Mattis dementia scale (/144) & $140.9(2.9)$ & $118.7(15.5)$ \\
\hline Educational level ${ }^{1}$ & $11.6(3.2)$ & $11.6(4.2)$ \\
\hline GDS scale, total score & $2.6(1.8)$ & $4.6(4.5)$ \\
\hline HAD scale, total score & $11.2(6.1)$ & $14.4(12)$ \\
\hline HAD scale, anxiety score & $7.4(3.7)$ & $7.8(5.3)$ \\
\hline HAD scale, depression score & $3.9(3)$ & $3.9(6.9)$ \\
\hline
\end{tabular}

${ }^{1}$ Number of schooling years; GDS = Global Deterioration scale (Yesavage et al., 1983); HAD = Hospital and Depression scale (Zigmond \& Snaith, 1983) 
Table 2. Cognitive performance of the two groups of participants on the word stem completion task

\begin{tabular}{|c|c|c|c|c|c|c|}
\hline \multicolumn{7}{|c|}{$\begin{array}{l}\text { Mean probabilities of completing stems with the targe } \\
\text { condition at each lag interval ( } 0 \text { vs. } 3 \text { vs. } 12 \text { ) }\end{array}$} \\
\hline 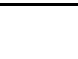 & \multicolumn{3}{|c|}{ Inclusion } & \multicolumn{3}{|c|}{ Exclusion } \\
\hline & Lag 0 & Lag 3 & Lag 12 & Lag 0 & Lag 3 & Lag 12 \\
\hline CS & $0.95(0.10)$ & $0.71(0.19)$ & $0.54(0.20)$ & $0.01(0.04)$ & $0.17(0.13)$ & $0.19(0.11)$ \\
\hline FTD & $0.83(0.20)$ & $0.46(0.19)$ & $0.28(0.20)$ & $0.09(0.22)$ & $0.31(0.15)$ & $0.35(0.17)$ \\
\hline \multicolumn{7}{|c|}{ Estimates of controlled and automatic memory processes at each lag interval ( 0 vs. 3 vs. 12) } \\
\hline & \multicolumn{3}{|c|}{ Controlled processes } & \multicolumn{3}{|c|}{ Automatic processes } \\
\hline CS & $0.93(0.10)$ & $0.54(0.26)$ & $0.34(0.26)$ & - & $0.36(0.22)$ & $0.29(0.13)$ \\
\hline FTD & $0.74(0.33)$ & $0.18(0.22)$ & $0.03(0.12)$ & - & $0.37(0.13)$ & $0.33(0.15)$ \\
\hline
\end{tabular}

Lag $=$ number of interfering trigrams between similar stems; $C S=$ control subjects, FTD $=$ bvFTD patients; Standard deviations appear in parentheses 
Table 3. Cognitive performance of FTD and control subjects on tasks tapping different executive processes

\begin{tabular}{|c|c|c|c|}
\hline & Control subjects & FTD patients & \\
\hline \multicolumn{4}{|l|}{ Stroop task } \\
\hline Interference RTs (sec.) & $132(44)$ & $171(54)$ & $\begin{array}{l}F(1,28)=4.47, p<0.05 \\
\text { partial ES }=0.14\end{array}$ \\
\hline Interference errors & $2.05(2.04)$ & $5.20(6.14)$ & $\begin{array}{l}F(1,28)=4.42, p<0.05, \\
\text { partial ES }=0.14\end{array}$ \\
\hline \multicolumn{4}{|l|}{ Hayling task } \\
\hline $\begin{array}{l}\text { Initiation condition } \\
\text { (RTs, sec.) }\end{array}$ & $1.04(0.70)$ & $1.85(1.25)$ & $\begin{array}{l}F(1,30)=8.65, p<0.01 \\
\text { partial } E S=0.22\end{array}$ \\
\hline $\begin{array}{l}\text { Suppression condition } \\
\text { (RTs, sec) }\end{array}$ & $4.84(2.29)$ & $4.77(2.10)$ & $\begin{array}{l}F(1,30)=0.006, p>0.5, \\
\text { partial ES }=0.0001\end{array}$ \\
\hline $\begin{array}{l}\text { Suppression condition } \\
\text { (semantic relatedness) }\end{array}$ & $5.60(2.21)$ & $20.21(9.48)$ & $\begin{array}{l}F(1,30)=44.44, p<0.0001 \\
\text { partial ES }=0.60\end{array}$ \\
\hline \multicolumn{4}{|l|}{ Verbal fluency } \\
\hline Semantic & $27.70(7.42)$ & $16.50(6.11)$ & $\begin{array}{l}F(1,30)=15.13, p<0.0001 \\
\text { partial } E S=0.33\end{array}$ \\
\hline Phonological & $24.80(6.03)$ & $11.25(5.83)$ & $\begin{array}{l}F(1,30)=43.72, p<0.0001 \\
\text { partial } E S=0.59\end{array}$ \\
\hline \multicolumn{4}{|l|}{ Trail Making Test } \\
\hline Part A (RTs) & $39.61(11.77)$ & $70.00(32.40)$ & \multirow{2}{*}{$\begin{array}{l}\text { Interaction effect: } \\
F(1,28)=7.41, p<0.05, \\
\text { partial ES }=0.21\end{array}$} \\
\hline Part B (RTs) & $94.53(36.93)$ & $161.20(52.12)$ & \\
\hline Part B (errors) & $0.20(0.52)$ & $2.10(2.33)$ & $\begin{array}{l}F(1,28)=12.46, p<0.005 \\
\text { partial ES }=0.31\end{array}$ \\
\hline \multicolumn{4}{|l|}{$\begin{array}{l}\text { The delayed } \\
\text { alternation task }\end{array}$} \\
\hline Total performance & $41.40(8.25)$ & $31.17(5.07)$ & $\begin{array}{l}F(1,30)=15.41, p<0.0005 \\
\text { partial ES }=0.33\end{array}$ \\
\hline
\end{tabular}




\begin{tabular}{|l|c|c|l|}
\hline The Brixton task & & & \\
\hline Total performance & $41.30(5.51)$ & $29.17(10.94)$ & $\begin{array}{l}F(1,30)=17.49, p<0.0005, \\
\text { partial ES }=0.37\end{array}$ \\
\hline Type 1 errors & $3.90(2.21)$ & $4.17(2.52)$ & $\begin{array}{l}F(1,30)=0.10, p>0.5, \text { partial } \\
E S=0.003\end{array}$ \\
\hline Type 2 errors & $6.75(4.42)$ & $8.17(4.76)$ & $\begin{array}{l}F(1,30)=0.73, p>0.1, \text { partial } \\
E S=0.03\end{array}$ \\
\hline Type 3 errors & $3.05(1.93)$ & $13.50(12.48)$ & $\begin{array}{l}F(1,30)=13.77, p<0.001, \\
\text { partial } \mathrm{ES}=0.31\end{array}$ \\
\hline
\end{tabular}

Standard deviations appear in parentheses. RTs= response times. Hayling task: initiation condition = completion of the sentence with the missing word; suppression condition = completion of the sentence with an unrelated word. Verbal fluency: number of words generated in $120 \mathrm{sec}$. Trail Making test: Part A= connecting randomly located letters in the alphabetical order, Part $B=$ connecting alternately numbers and letters in their respective sequence. Brixton task: Type 1 errors $=$ perseverations at the stimulus-response or the set level, Type 2 errors = applications of other rules that were previously relevant, Type 3 errors $=$ responses which could not be included in first or second type since no apparent underlying rationale could be discovered. 
Table 4. Cognitive performance of FTD and control subjects on tasks requiring automatic processes

\begin{tabular}{|c|c|c|c|}
\hline & Control subjects & FTD patients & \\
\hline The phonological loop & & & \\
\hline $\begin{array}{l}\text { Short and phonologically } \\
\text { dissimilar words span }\end{array}$ & $4.55(0.69)$ & $4.08(1)$ & \multirow{3}{*}{$\begin{array}{l}\text { Interaction effects : } \\
\text { Articulatory rehearsal } \\
F(1,30)=0.52, p=0.47, \\
\text { partial } \mathrm{ES}=0.02 \\
\text { Phonological store: } \\
F(1,29)=1.10, p=0.30, \\
\text { partial } \mathrm{ES}=0.04\end{array}$} \\
\hline Long words span & $3.60(0.60)$ & $3.33(0.65)$ & \\
\hline $\begin{array}{l}\text { Phonologically similar words } \\
\text { span }\end{array}$ & $3.70(0.66)$ & $3.73(0.79)$ & \\
\hline \multicolumn{4}{|l|}{ Letter matching task } \\
\hline RTs (msec) & $878(226)$ & $1084(394)$ & $\begin{array}{l}F(1,28)=3.32, p=0.08 \\
\text { partial } E S=0.11\end{array}$ \\
\hline Total errors & $1.05(1.40)$ & $1.60(1.35)$ & $\begin{array}{l}F(1,28)=1.06, p=03.1, \\
\text { partial } E S=0.04\end{array}$ \\
\hline \multicolumn{4}{|l|}{ Stroop task } \\
\hline Naming RTs & $70.30(16.94)$ & $83.09(23.18)$ & $\begin{array}{l}F(1,29)=3.11, p=0.09 \\
\text { partial } E S=0.10\end{array}$ \\
\hline Naming errors & $0.80(1.28)$ & $1.00(1.41)$ & $\begin{array}{l}F(1,29)=0.16, p=0.69 \\
\text { partial } E S=0.005\end{array}$ \\
\hline Reading RTs & $46.80(8.90)$ & $54.91(13.98)$ & $\begin{array}{l}F(1,29)=4.04, p=0.05, \\
\text { partial } E S=0.12\end{array}$ \\
\hline Reading errors & $0.00(0)$ & $0.10(0.44)$ & \\
\hline
\end{tabular}

Standard deviations appear in parentheses. RTs $=$ response times. Span size $=$ the longest sequence in which at least two sequences had been correctly recalled 
Table 5. Comparison between ratings for premorbid and current behaviour (FTD patients only) on total score and internalizing/externalizing subscales of the ISPC.

\begin{tabular}{|l|l|l|l|}
\hline ISPC dimensions & Premorbid level & Current level & \\
\hline Total score & $68.36(15.17)$ & $126.54(37.66)$ & $\mathrm{Z}=2.93, \mathrm{p}<0.005$ \\
\hline ISPC-Externalizing & $19.09(6.68)$ & $33.09(13.77)$ & $\mathrm{Z}=2.65, \mathrm{p}<0.01$ \\
\hline ISPC-Internalizing & $6.09(3.18)$ & $14.09(5.82)$ & $\mathrm{Z}=2.93, \mathrm{p}<0.005$ \\
\hline
\end{tabular}

Note. The importance of behavioural changes was assessed by summing the absolute value of the differential score («now - before ») for each dimension. Standard deviations appear in parentheses 